\title{
Spontaneous transitions to focal-onset epileptic seizures: A dynamical study
}

Cite as: Chaos 30, 103114 (2020); https://doi.org/10.1063/5.0021693

Submitted: 13 July 2020. Accepted: 09 September 2020 . Published Online: 14 October 2020

(iD) Liyuan Zhang, Qingyun Wang, and (i) Gerold Baier

\section{COLLECTIONS}

Paper published as part of the special topic on Dynamical Disease: A Translational Perspective
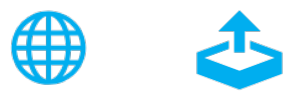

\section{ARTICLES YOU MAY BE INTERESTED IN}

Chaos in Cartan foliations

Chaos: An Interdisciplinary Journal of Nonlinear Science 30, 103116 (2020); https:// doi.org/10.1063/5.0021596

Bifurcations and chaos in a Lorenz-like pilot-wave system

Chaos: An Interdisciplinary Journal of Nonlinear Science 30, 103115 (2020); https:// doi.org/10.1063/5.0020775

\section{Assessing observability of chaotic systems using Delay Differential Analysis}

Chaos: An Interdisciplinary Journal of Nonlinear Science 30, 103113 (2020); https://

doi.org/10.1063/5.0015533

\section{AIP Advances}




\title{
Spontaneous transitions to focal-onset epileptic seizures: A dynamical study
}

\author{
Cite as: Chaos 30, 103114 (2020); doi: 10.1063/5.0021693 \\ Submitted: 13 July 2020 . Accepted: 9 September 2020 . \\ Published Online: 14 October 2020

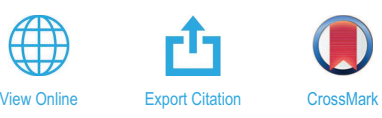

Liyuan Zhang, ${ }^{1,2}$ (D) Qingyun Wang, ${ }^{3}$ and Gerold Baier ${ }^{4, a)}$ (i)

\section{AFFILIATIONS}

${ }^{1}$ Department of Biomedical Engineering, Faculty of Environment and Life, Beijing University of Technology, 100124 Beijing, China ${ }^{2}$ Beijing International Science and Technology Cooperation Base for Intelligent Physiological Measurement and Clinical

Transformation, 100124 Beijing, China

${ }^{3}$ Department of Dynamics and Control, Beihang University, 100191 Beijing, China

${ }^{4}$ Cell and Developmental Biology, University College London, London WC1E 6BT, United Kingdom

Note: This paper is part of the Focus Issue on Dynamical Disease: A Translational Perspective.

author to whom correspondence should be addressed: g.baier@ucl.ac.uk

\begin{abstract}
Given the complex temporal evolution of epileptic seizures, understanding their dynamic nature might be beneficial for clinical diagnosis and treatment. Yet, the mechanisms behind, for instance, the onset of seizures are still unknown. According to an existing classification, two basic types of dynamic onset patterns plus a number of more complex onset waveforms can be distinguished. Here, we introduce a basic three-variable model with two time scales to study potential mechanisms of spontaneous seizure onset. We expand the model to demonstrate how coupling of oscillators leads to more complex seizure onset waveforms. Finally, we test the response to pulse perturbation as a potential biomarker of interictal changes.
\end{abstract}

Published under license by AIP Publishing. https://doi.org/10.1063/5.0021693

Focal epileptic seizures are characterized by complex spatiotemporal rhythms of electric brain activity. Phenomenologically, there are different types of rhythms, namely, fast-small oscillations, slow-large spiking, and complex waveforms of the voltage. The dynamics of these types are, however, still poorly understood and their clinical characterization is, therefore, mostly descriptive. We use computational modeling of macro-level electrical brain activity to study the spontaneous onset and evolution of major clinical seizure rhythms. Looking at principal models of one and two coupled oscillators with a slow driving population, we show the dynamical characteristics and relationships between basic seizure onset types. In addition, we show that pulse perturbations of background activity can serve as a biomarker for seizure onset.

\section{INTRODUCTION}

Epilepsy is a neurological disorder that is characterized by recurrent abnormal seizures discharges. According to the classification and terminology revised by the International League Against Epilepsy (ILAE), focal epilepsy is defined as seizures appearing within networks limited to one hemisphere and either dispersedly localized or globally distributed. ${ }^{1}$ The onset patterns of focal epilepsies have received more attention ${ }^{2,3}$ because understanding the onset can lay the foundation of revealing the nature of seizures in drug-resistant focal epilepies. In the previous studies, the fastsmall amplitude onset and the slow-large amplitude onset were widely investigated from a clinical point of view ${ }^{4-6}$ and also studied using computational simulations. ${ }^{7}$ However, complex waveform onsets, including sharp activity, spike-and-wave, burst of highamplitude polyspikes, burst suppression, and delta brush, are also seen and may result from different pathological substrates. ${ }^{8}$ Furthermore, studies have demonstrated that focal epilepsy genesis may refer to specific cortical and subcortical networks., can detect anatomical or functional abnormalities in focal epilepsy, the dynamic mechanisms that govern seizures are not well understood. Thus, a better understanding of the mechanisms underlying seizures and specifically seizure onset is of significance for advances in accurate diagnosis. 
Dynamical characteristics of focal epilepsy are commonly investigated in animal models. However, mathematical models provide a safe complementary way of study. There are two main levels of description of computational models that describe transitions to seizure: at the microscopic level, detailed neuronal network models (e.g., the Hodgkin-Huxley neuronal model) constructed with principal neurons and interneurons connected by means of chemical synapses or gap junctions, can be used to build networks which produce epileptiform activities. ${ }^{11}$ At the macro-level, neural field models can describe the spatiotemporal evolutions of coupled populations on the macro-scale relating to, e.g., electrocorticography (ECoG) or stereo-encephalography (SEEG). ${ }^{12}$ For example, the dynamic mechanisms of two different onset patterns of focal seizures were investigated by means of networks of Wilson-Cowan neural field oscillators.

In terms of dynamical systems, epileptic seizure discharges can be viewed as instances of bursting. Generally, bursting discharges are induced by fast slow dynamics. ${ }^{14}$ At the scale of a mean field model, e.g., Liley and Walsh ${ }^{15}$ established a fast-slow model by adding slow populations that led to bursting activity. ${ }^{16}$ Therein, they hypothesized that the addition of a slow population could represent superimposed effects of intracortical, intercortical, and subcortical systems. Specifically, they hypothesized that the slow system might originate from thalamo-cortical feedback, modulations in the conduction specialties of the long-range corticocortical fiber system, or slow changes due to synaptic activities, e.g., uptake (depletion) process by glial cells and reuptake (restitution) processes. This is further corroborated by investigations on the role of astrocyte cells (a subtype of glia). ${ }^{17,18}$ Motivated by the idea that spontaneous epileptic seizures can result from the driving by a slow underlying process, we first present a principal threevariable model of spontaneous seizure transitions, then expand it to a model of coupled oscillators with a slow driving population, ${ }^{19,20}$ and show the resulting relationships between the basic focal-onset types and more complex types of dynamics. Finally, we look at the response of the temporally evolving interictal state to repetitive pulse perturbations.

\section{MODELS}

\section{A. The basic three-variable model}

Epileptic seizure dynamics can be viewed as repeated transitions of macroscopic electric brain activity between a quiescent (non-epileptic) and a rhythmic (epileptic) state. We, therefore, start with a three-variable system of coupled differential equations to create spontaneous switching between a quiescent background and oscillatory dynamics. The model is of the type that has been used in mean field modeling in many previous studies, closely related to the Amari model ${ }^{20}$ and the Wilson-Cowan model. ${ }^{7}$ The basic construction is an excitatory population $E X$, which positively impacts an inhibitory population IN. The inhibitory population in turn negatively impacts the excitatory population. Given specific choices of parameters, the model has bifurcations without (supercritical Hopf and saddle-node on invariant cycle, SNIC) or with (subcritical Hopf and homoclinic bifurcation) the presence of bistability. ${ }^{2}$ These codimension 1 bifurcations represent the basic transitions from fixed point to oscillatory behavior in two-variable deterministic model systems. Together with the four codimension 1 bifurcations that can lead to the disappearance of an attracting limit cycle in the absence or presence of bistability, they combine to yield a set of 16 possible type of bursting under slow variation of a model parameter.

A common assumption about the onset of focal epileptic seizures is that a slow shift in one of the parameters drives a transition in and out of the oscillatory state, thus accounting for the onset and offset of seizures. The nature of the assumed shifting parameter is not known in humans but some evidence from animal models points to slow changes in the extracellular space surrounding the neural population. From a dynamical systems point of view, a transition between a quiescent and an oscillatory state can occur spontaneously if the $E X-I N$ oscillator is connected to a very slow population in a feedback loop. ${ }^{21}$ An equation of such a model of spontaneous transitions was given in Ref. 20 but not studied in any detail. Here, we describe its properties as a candidate for an extended network model of focal-seizure onset. The differential equations are as follows:

$$
\begin{aligned}
\frac{d E X}{d t} & =\tau_{e x}\left(h_{\mathrm{ex}}-E X+C 1 f[E X]-\mathrm{C} 2 f[I N]-C U 1 f[U L]\right), \\
\frac{d I N}{d t} & =\tau_{\mathrm{in}}\left(h_{\mathrm{in}}-I N+C 3 E X\right), \\
\frac{d U L}{d t} & =\tau_{u l}\left(h_{u l}-U L+\mathrm{C} 1 \mathrm{U} f[E X]\right),
\end{aligned}
$$

where $E X$ represents the excitatory population and $I N$ the inhibitory population. $U L$ denotes a population that integrates inputs and generates slowly varying feedback. Here, it receives input from the $E X$ population and feeds back to $E X$ with coupling achieved through a sigmoidal activation function. The input to the inhibitory variable is using a linear term similar to a previous approach. ${ }^{22} f[x]$ is a sigmoidal activation function defined as $f[x]=\frac{1}{1+\varepsilon^{-x}}$ where $\mathrm{x}=E X, I N$, and $U L$, respectively, and $\varepsilon=1000$. The model scheme is shown in Fig. 1. We consider the constant input $h_{\mathrm{ex}}$ as bifurcation parameter.

\section{B. Two coupled oscillators modulated by a common ultraslow population}

We extend the above model to a system with two coupled oscillators driven by a joint ultraslow population. Each oscillator has an excitatory and an inhibitory population (EX1, IN1 and $E X 2, I N 2$, respectively) as before. There is coupling between them which is bi-directional but typically with different coupling strengths. Both oscillators feed into an ultraslow population. In contrast to previous approaches, ${ }^{20}$ we assume the ultraslow population to interact reciprocally with both oscillators, i.e., it plays a global role in driving the model into and out of an oscillatory rhythm. The full set of interactions between the populations of the oscillators and the ultraslow population is shown schematically in Fig. 2. 


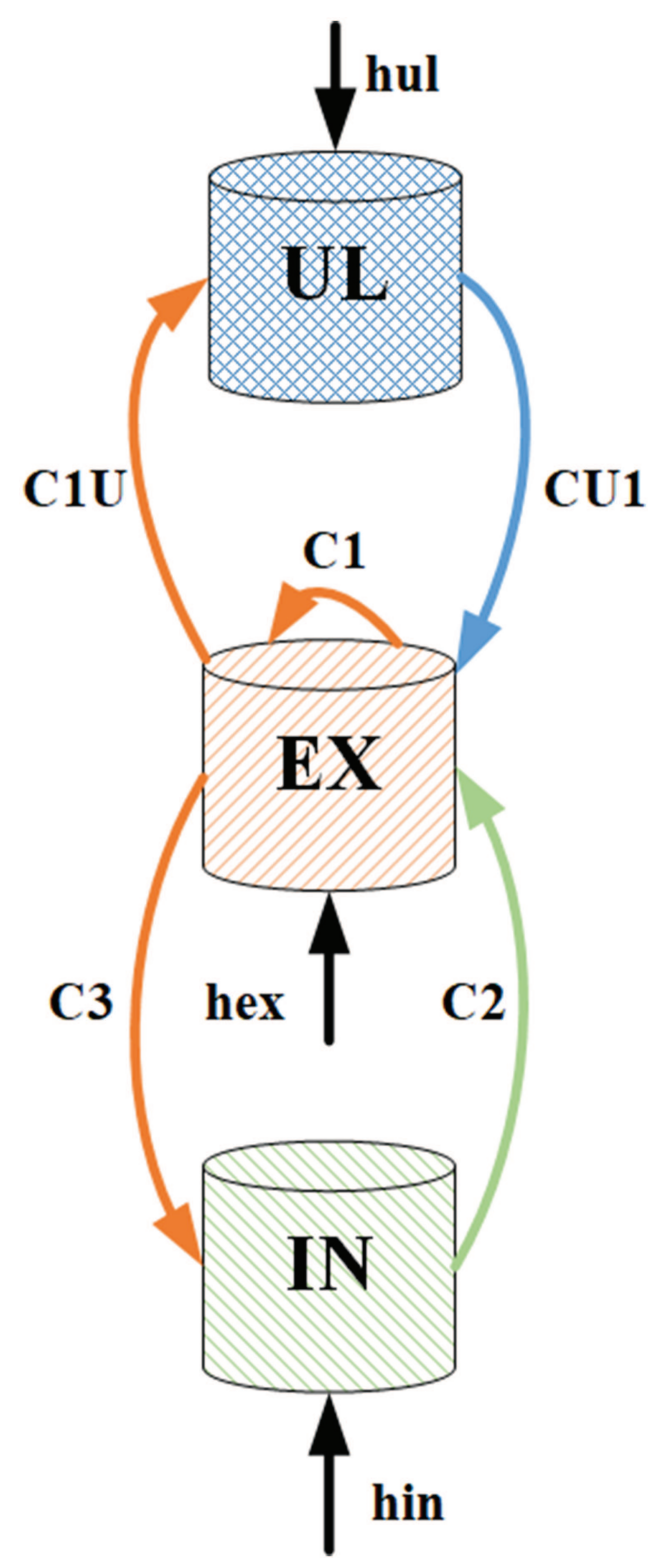

FIG. 1. Configuration of the basic three variables, one excitatory population $(E X)$, one inhibitory population $(I N)$, and one population can produce slowly varying output (UL). The $h$-parameters are constant inputs. $h_{\text {ex }}$ is the input of $E X, h_{\text {in }}$ is for $I N$, and $h_{u l}$ is for UL. The $C$-labels are connection parameters. $C 1$ is the self-connection parameter of $E X, C 2$ is the connection parameter from $I N$ to $E X$, $C 3$ is from $E X$ to $I N, C 1 U$ is from $E X$ to $U L$, and $C U 1$ is from $U L$ to $E X$.

The equations to describe the temporal evolution of the twooscillator model with slow modulation are

$$
\begin{aligned}
\frac{d E X 1}{d t}= & \tau_{e x 1}\left(h_{e x 1}-E X 1+C 1 f[E X 1]-C 2 f[I N 1]\right. \\
& +C 21 f[E X 2]-C U 1 f[U L])
\end{aligned}
$$

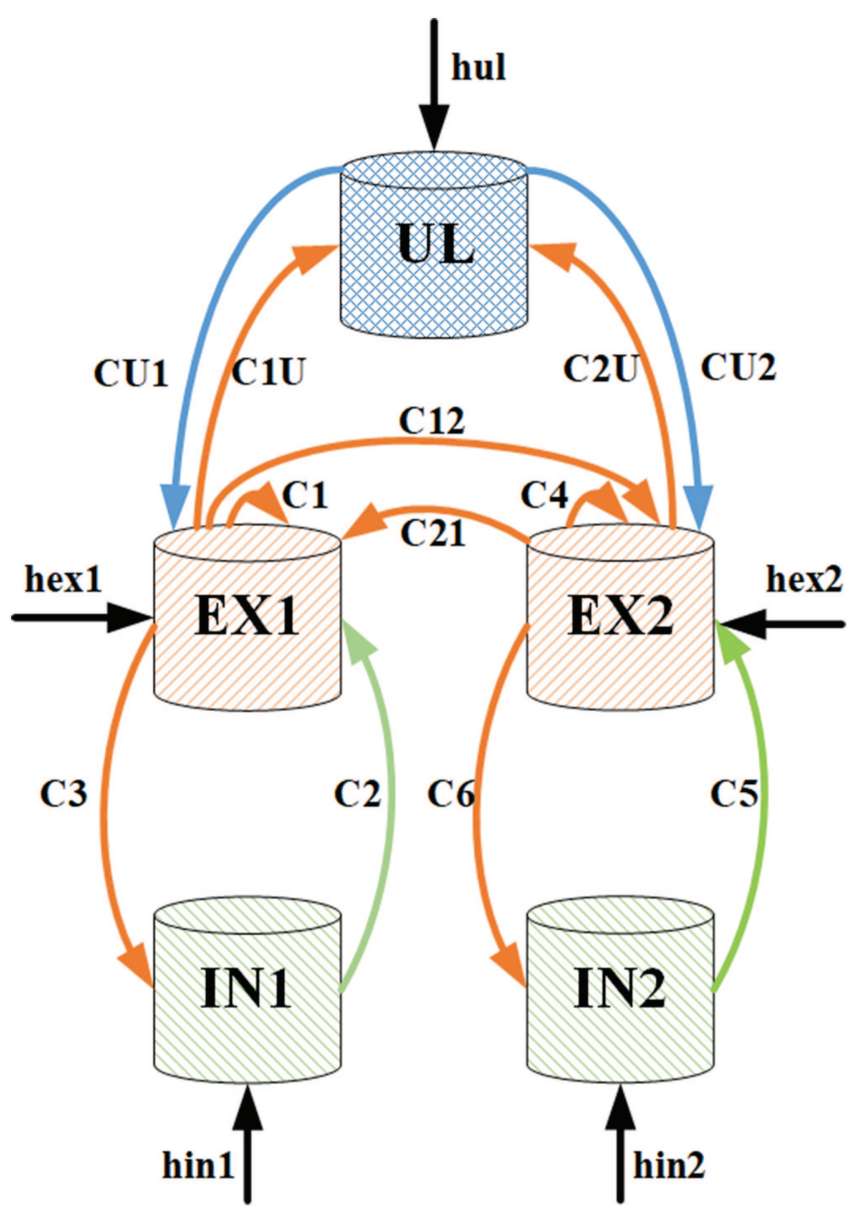

FIG. 2. Diagram of a model of two coupled oscillators modulated by a common ultraslow population. Excitatory populations are represented by EX1 and EX2, and inhibitory populations are IN1 and IN2. UL is the population that can generate slow output. The $C$-labels denote connection parameters. $C 1$ and $C 4$ are the self-connection parameters of $E X 1$ and $E X 2$, respectively. $C 2$ is the connection parameter from IN1 to EX1,C3 is from EX1 to IN1, C1U is from EX1 to $U L$ and CU1 is from UL to EX1.C5 is the connection parameter from IN2 to EX2, C6 is from EX2 to IN2, C2U is from EX2 to UL, and CU2 is from UL to EX2. The $h$-labels are constant external inputs. $h_{\mathrm{ex} 1}$ and $h_{\mathrm{ex} 2}$ are the inputs of EX1 and $E X 2$, respectively. $h_{i n 1}$ and $h_{i n 2}$ are for IN1 and IN2, respectively. $h_{u l}$ is for UL.

$$
\begin{aligned}
\frac{d I N 1}{d t}= & \tau_{i n 1}\left(h_{i n 1}-I N 1+C 3 E X 1\right), \\
\frac{d E X 2}{d t}= & \tau_{e x 2}\left(h_{e x 2}-E X 2+C 4 f[E X 2]-C 5 f[I N 2]\right. \\
& +C 12 f[E X 1]-C U 2 f[U L]), \\
\frac{d I N 2}{d t}= & \tau_{i n 2}\left(h_{i n 2}-I N 2+C 6 E X 2\right), \\
\frac{d U L}{d t}= & \tau_{u l}\left(h_{u l}-U L+C 1 \mathrm{U} f[E X 1]+C 2 U f[E X 2]\right) .
\end{aligned}
$$


According to the original paper of Wilson and Cowan, ${ }^{23}$ the time constant $\tau$ is the range for the delays associated with the propagation of postsynaptic potentials from the dendrites of a neuron to the axon hillock. In our study, time scales are denoted by the $\tau_{i}$ parameters. We pick time scale parameters as $\tau_{e x 1}=\tau_{i n 1}=1$ and $\tau_{e x 2}=\tau_{\text {in2 }}=1.1$ for similar but not identical time scales of the oscillators (to avoid unrealistic symmetry) and $\tau_{e x 1}=\tau_{i n 1}=1$ and $\tau_{e x 2}$ $=\tau_{\text {in } 2}=5$ for different time scales of the oscillators. We consider the two coupling constants $C 12$ and $C 21$ as bifurcation parameters.

Simulations were done in MATLAB and confirmed independently with a different integration routine in Python.

\section{RESULTS}

\section{A. Dynamical analysis of the three-variable model}

A summary of exemplary dynamics of the three-variable model is provided in Fig. 3. Figure 3(a1) is the bifurcation diagram as a function of input parameter hex. Two regions of fixed point dynamics enclose a region of spontaneous oscillations. The oscillatory region is divided into a region of bursting $\left(-0.6 \leq h_{e x} \leq-0.2\right)$ and a region of small-amplitude periodic oscillations that vanish in a supercritical Hopf bifurcation at around hex $\approx 0.18$. We regard the fixed point on the left-hand side of the diagram as our model for the stable background state and the bursting region as representing a state of spontaneous transition to epileptiform brain dynamics. The time series within the bursting region is a composite of a lower branch fixed point (representing non-seizure dynamics) and a slow transition from non-oscillatory to oscillatory dynamics on an upper branch. The jump from the lower quiescent state to the second, upper state is in agreement with a saddle-node bifurcation of the oscillatory subsystem $E X-I N$. From that "upper" state, we see a small drop to a second quiescent state, which appears to be a stable focus (it is approached by small damped oscillations at constant frequency). The next is an instability of that "focus" leading to the appearance of small-fast oscillations. The increase in amplitude is at least compatible with a subcritical Hopf bifurcation transiting to a pre-existing large cycle with lower frequency in a hypothetic time scale separated system. Eventually, the oscillations terminate with what appears to be a saddle-homoclinic orbit to the (already existing) "lower" quiescent state. The zoom of the time series around oscillatory onset in Fig. 3(b1) shows the small drop at the higher branch to an oscillatory onset with initially very small amplitude and high frequency followed by a continuous increase in amplitude.

Figure 3(a2) is the bifurcation diagram as a function of parameter hex at a parameter set that differs only in the setting of the input and time scale of the inhibitory population. We also find a bursting region $\left(-0.59 \leq h_{e x} \leq-0.49\right)$ enclosed by a fixed point for small values of hex and an oscillatory region for large values of hex. In the bursting region, the oscillatory onset shows repetitive large amplitude and low frequency spiking. The lower quiescent state transits to oscillations via what would be a SNIC bifurcation of the fast subsystem. This leads to the sudden appearance of large spikes at an initially low frequency [Fig. 3(b2)]. As the oscillation evolves farther away from the transition point, the frequency of the large spiking increases, while the amplitude is preserved. The end of the oscillatory phase is compatible with a saddle-homoclinic orbit as an abrupt stop of the spiking and a drop to a non-oscillatory state, which already exists. In the terminology of Izhikevich, this would represent a "circle / homoclinic bursting." 21

Both scenarios are at least consistent with previous studies of the seizure onset and offset as cases of embedded low-dimensional bifurcations..$^{24,25}$ However, this is only an approximation based on the fast/slow separation $\left(\tau_{u l} \rightarrow \tau_{e x} / \infty\right)$, whereas the full model is
(A1)

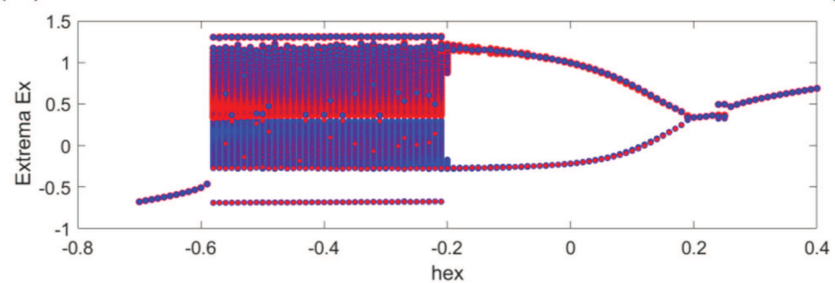

(B1)

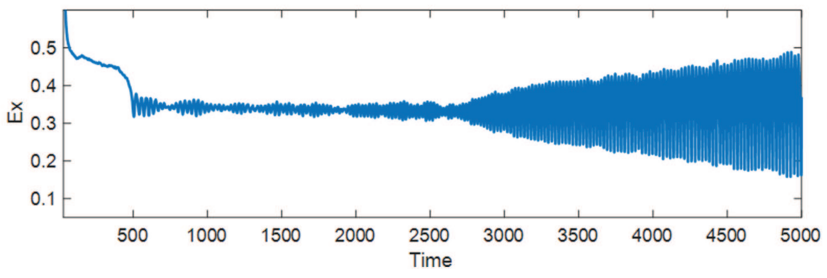

(A2)

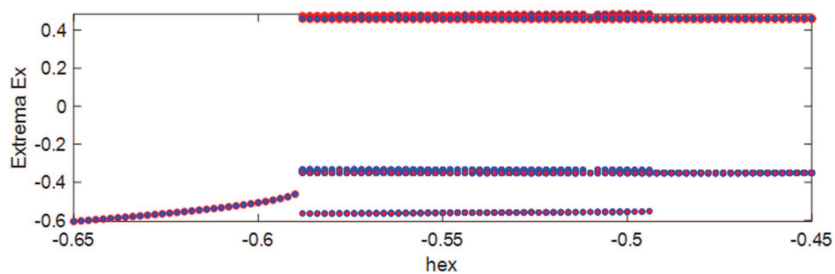

(B2)

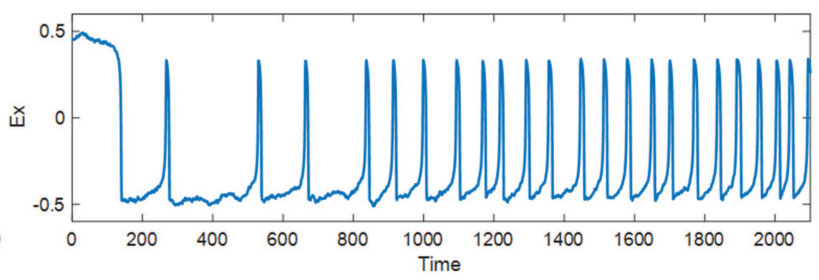

FIG. 3. Bifurcation diagram and time series of the simulated "seizure" onset in Eq. (1). (a1) and (a2): Bifurcation diagrams of fast-small onset and slow-large onset, respectively. Parameters (a1): $C 1=3.5, C 2=2.3, C 3=6, C U 1=C 1 U=1, \tau_{\mathrm{ex}}=2, \tau_{\mathrm{in}}=2, \tau_{u l}=\tau_{\mathrm{ex}} / 1000, h_{\mathrm{in}}=-1.5$, and $h_{u l}=-0.7$. Parameters (a2): same as (a1) except $\tau_{\text {in }}=5.5, h_{\text {in }}=-0.2$. (b1) and (b2): Zooms of the corresponding time series. Input of excitatory population in (b1) is $h_{\mathrm{ex}}=-0.503$, and $h_{\mathrm{ex}}=-0.5535$ in (b2). Other parameters as in (a1) and (a2), respectively. For the time series, the time scale of the ultraslow population was set to $\tau_{u l}=\tau_{\mathrm{ex}} / 5000$ to simulate a long quiescent period and Gaussian white noise with mean 0 and variance 0.1 added. 
nonlinear and parameters are not set within strict validity of this simplification. The two parameter settings are such that they are compatible with a codimension 1 situation in the two-variable subsystem. Thus, if, e.g., a linear interpolation was made between the parameters, they would pass the vicinity of a codimension 2 point in that susbsystem. Note, however, that even this model already has a parameter space that is difficult to treat analytically for all possible bifurcations and that the model structure might violate the assumptions of an independently varied slow parameter, i.e., the ultraslow variable is not independent of the happenings in the oscillator.

\section{B. Spontaneous transitions in the extended two-coupled oscillator model}

Based on previous studies of the dynamic transitions in focalonset epileptic seizures, ${ }^{26}$ we look at the influence of parameter changes in a model of two coupled oscillators with similar and different time scale parameters, respectively. The reason for this is that epileptic seizure rhythms are not restricted to a single macroscopic time scale and that in many instances the seizure onset and evolution is not restricted to either fast or slow oscillations but mixed mode oscillations are found. ${ }^{8}$ The full parameter space of the 5 variable model is too high-dimensional to be studied in detail; therefore, only representative parameter sets are considered. The local excitability in oscillators 1 and 2 is set to hex $1=$ hex $2=-0.55$, respectively. These correspond to a value where the oscillators are in the background state but close to the bifurcation leading to oscillatory dynamics. For different values of parameter $C 12$, the coupling from oscillator 1 to oscillator 2, we scan parameter $C 21$, the coupling from oscillator 2 to oscillator 1 . These are the parameters to study both coupling and difference in coupling strength. The resulting dynamics are then studied further for the case of similar time scale parameters, respectively.

Figure 4 illustrates the bifurcations as displayed by extrema of oscillator 1 as a function of parameter $C 21$ (for $C 2112=1.5$ ) with similar time scales of the oscillators; time plots corresponding to both oscillators; and state space portraits for different values of C21. The bifurcation diagram in Fig. 4(a) shows that the model displays a stable steady state for coupling below $C 21 \approx 0.45$. There is a broad central region of spontaneous transitions between quiescent background and oscillations between $C 21 \approx 0.65$ and $C 21 \approx 3.7$. For strong coupling, $C 21>3.7$, we find permanent oscillatory activity. The central region is considered a model for spontaneously induced rhythmic activity corresponding to epileptic seizure dynamics.

For $C 21 \approx 0.73$ [Figs. 4(b1), 4(b2), and 4(c1)], it can be seen how the model spontaneously enters and exits oscillatory dynamics. The reason is that the quiescent background state has become unstable. The instability leads to a continuous slow decrease of the ultraslow population which results in a correspondingly slow increase of the excitatory and inhibitory variables of the two oscillators. This drives them across a threshold into oscillatory dynamics. At the switch to oscillations, the ultraslow population reverses and starts to continuously increase. That increase governs the evolution of the oscillatory dynamics until a second threshold is reached and the oscillation ends. The variables of the two oscillators resume a quasi-steady state value upon which the cycle starts from the beginning. The exact waveforms of the oscillatory parts vary from one burst to another, indicating that the overall attracting dynamics is quasiperiodic or weakly chaotic. The oscillatory period is initiated with two or three separated large spikes followed by an increase in frequency. This is reminiscent of the saddle-node on invariant cycle bifurcation that would be seen if the two oscillators were driven by a slow parameter increase in the absence of the ultraslow population. ${ }^{20}$ During the oscillatory part, the time series of the two oscillators share a common main frequency but the waveforms differ substantially. Looking closely, one can see a variation from a slower and larger oscillation to a faster smaller oscillation and back again before the rhythm terminates for both oscillators. The state space portrait in Fig. 4(c1) shows part of the underlying attractor dynamics stretched in the direction of the ultraslow population. During the pseudo-steady state period, the ultraslow population falls, and during the oscillatory period, it rises.

For $C 21 \approx 1.04$ [Figs. 4(b3), 4(b4), and 4(c2)], the time series of both oscillators in the oscillatory period start with slow activity which becomes continuously faster. The amplitude is modulated but regular until toward the end, the dynamics changes to irregular, slower and with larger amplitude. The state space portrait in Fig. 4(c2) shows the regular small oscillations forming a tube-like structure and the wider loops of the final part on top.

For $C 21 \approx 2.33$ [Figs. 4(b5), 4(b6), and 4(c3)], the switch from the pseudo-steady state leads to a long period of silence (a second, upper pseudo-steady state) before oscillatory activity sets in. The rhythm of both oscillators exhibits small-fast oscillatory activity with continuously increasing amplitude. There is a final more irregular and slow part which varies between instances of the seizure rhythm. Dynamically, this is reminiscent of a driving along a bistability of two steady states (the lower and the upper one) followed by a supercritical Hopf bifurcation of the upper steady state. The state space portrait in Fig. 4(c3) shows the slow branches of the two pseudosteady states and the fast transition between them as well as the oscillatory section for large values of the ultraslow population.

For $C 21=3$ [Figs. 4(b7), 4(b8), and 4(c4)], the time series are qualitatively similar to the case $C 21 \approx 2.33$, but the oscillatory period is longer and the frequency is higher, i.e., there is a notable increase in frequency in both oscillators.

At parameter settings in the oscillatory region near the onset $C 21 \approx 0.5$, the behavior is composed of single sharp spikes at very low frequency. In the presence of noise, this produces time series reminiscent of interictal epileptiform discharges.

To simulate interaction of oscillators with different time scales, we use $C 12=0.5$ and set the time scale parameter of oscillator 2 to be five times larger than that of oscillator 1. Figure 5 illustrates the bifurcations of the model, time series corresponding to the two oscillators, and state space portraits. For $C 21 \approx 1.63$ [Figs. 5(b1), 5(b2), and 5(c1)], the oscillatory period of oscillator 2 displays sharp oscillatory activity from beginning to the end with small modulations of the amplitude by the main slow frequency component of oscillator 1. Oscillator 1 has a large-slow oscillation of the relaxation type with fast oscillations imposed on the upper slow branch. As above, the details of the oscillations vary from one instance to another. The state space portrait shows that the combination of the fast and the slow component creates a shape reminiscent of a torus which is entered from one side (large values of EX1) and left from the other (low values of EX1). 
(A)
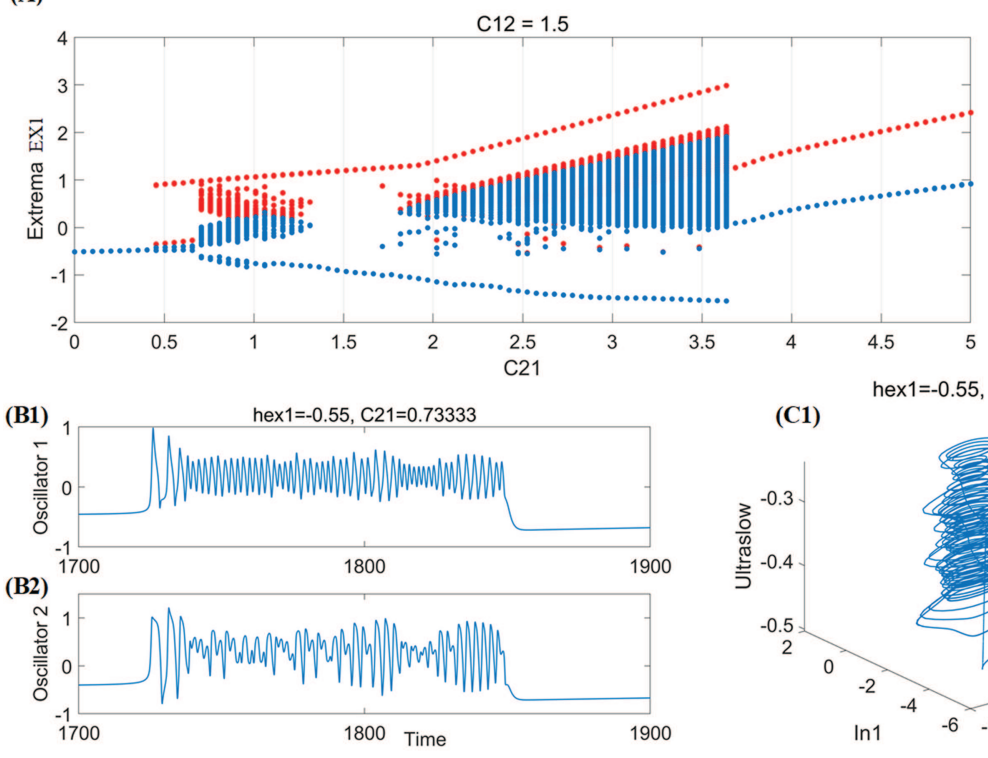

(B3)
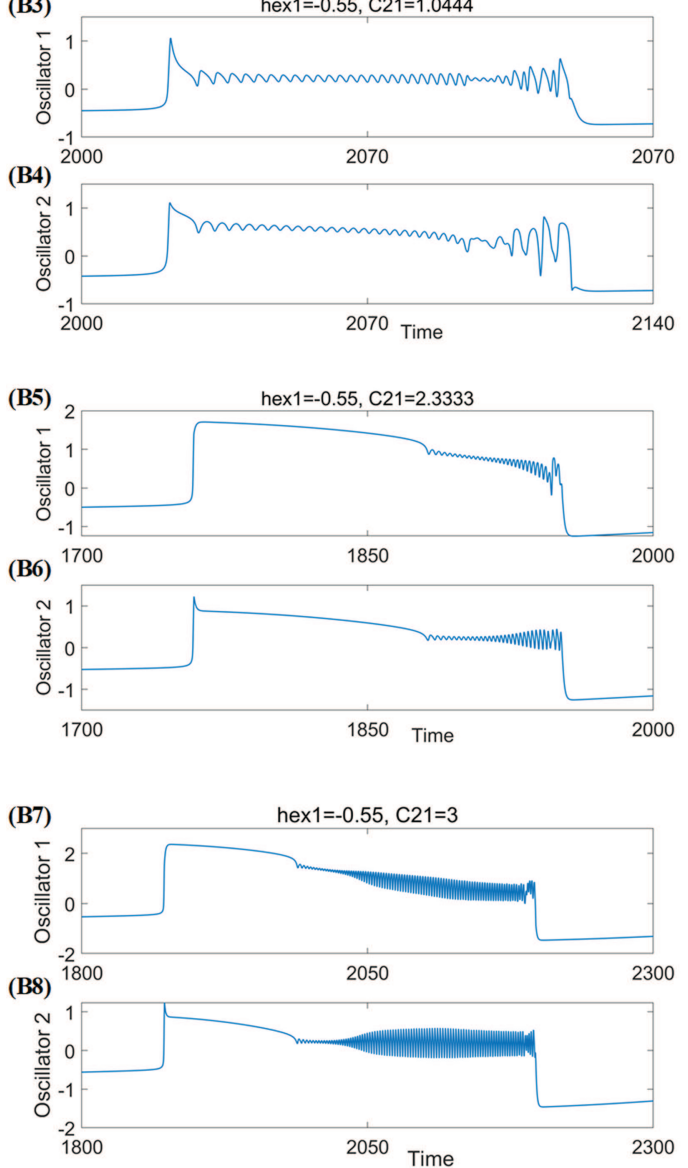

(C1)
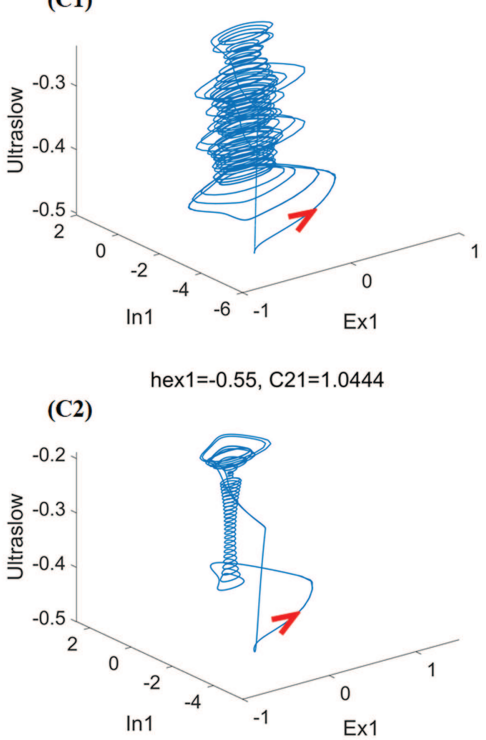

(C3) $\quad h e x 1=-0.55, C 21=2.3333$

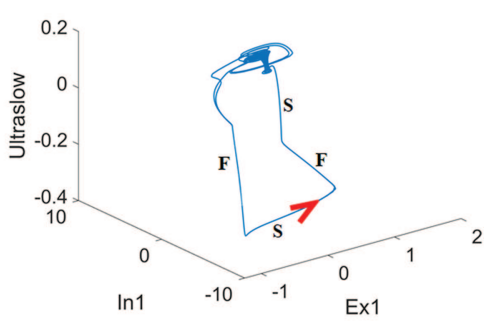

(C4)

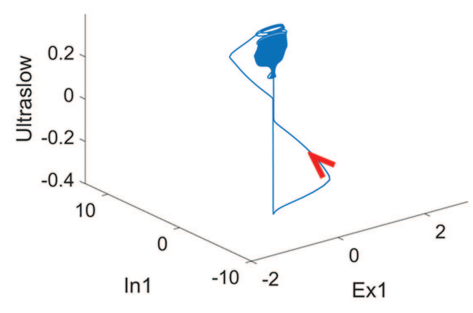

FIG. 4. Effect of coupling C21 on seizure onset and evolution for similar time scales and $\mathrm{C} 12=1.5$. (a) Bifurcation diagram showing extrema of oscillator 1 as a function of C21. Blue dots indicate either steady state or minima of Ex1 in the case of oscillatory dynamics. Red dots are the maxima. (b1) and (b2) are the time series of both oscillators for $\mathrm{C} 21 \approx 0.73$. (c1) is the pseudo-three-dimensional state space portraits with EX1, IN1 and the ultraslow population (direction indicated by red arrowhead). Following this arrangement, (b3), (b4), and (c2) are for C21 $\approx 1.04$, (b5), (b6), and (c3) are for $C 21 \approx 2.33$, and (b7), (b8), and (c4) are for $\mathrm{C} 21=3$. Fast $(\mathrm{F})$ and slow (S) branches of the trajectory are indicated in (c3). 
(A)

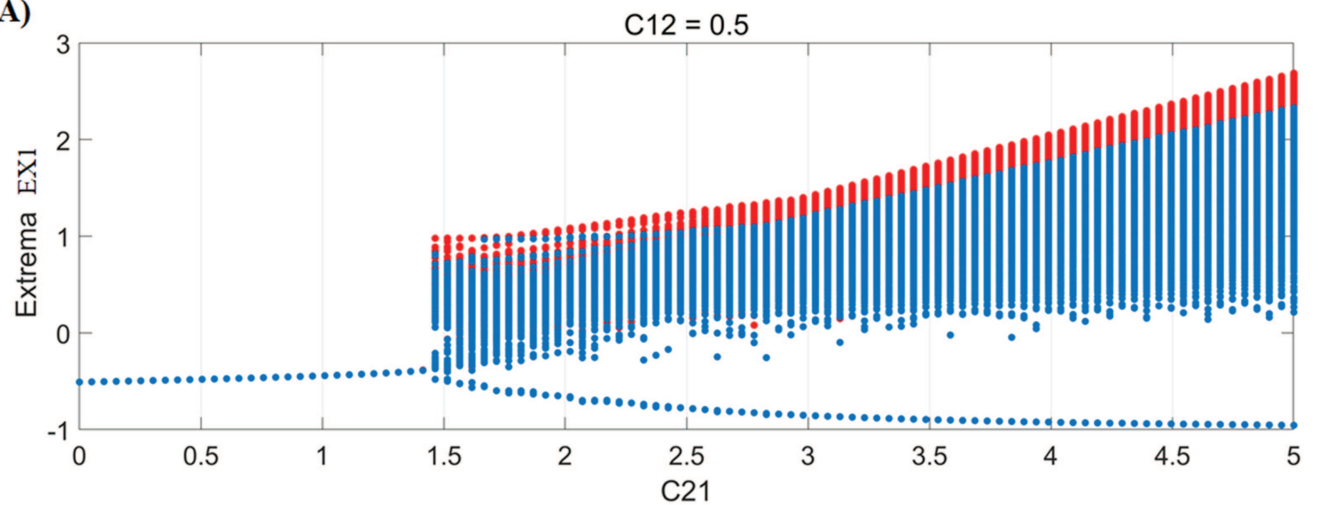

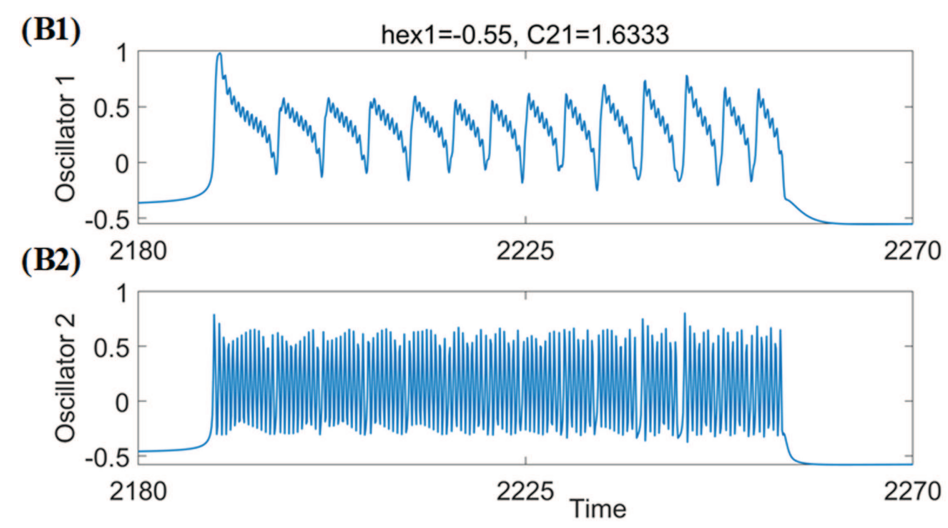

(B3)

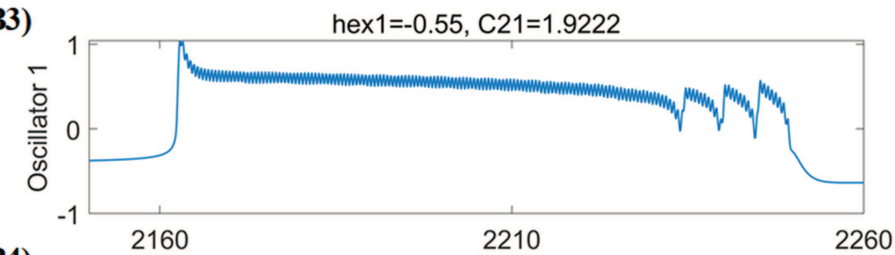

(B4)

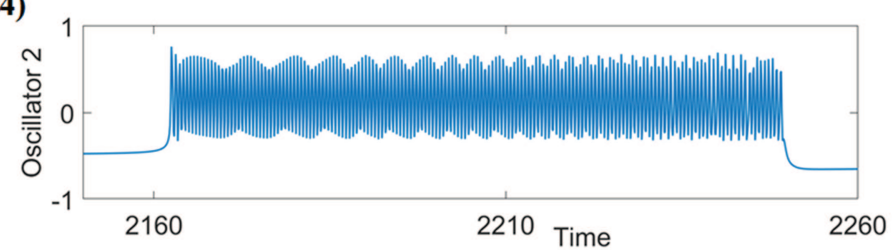

(C1)

hex1=-0.55, C21=1.6333

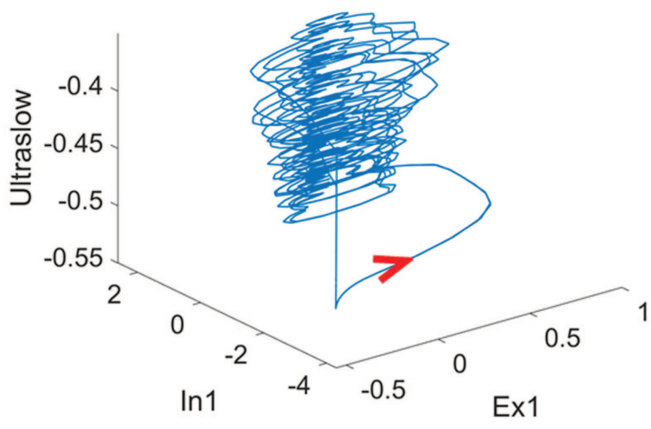

(C2)

hex1=-0.55, C21=1.9222

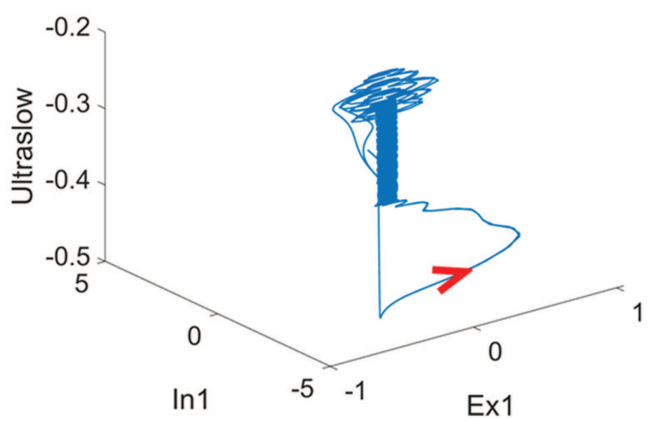

FIG. 5. Effect of $C 21$ on seizure onset for oscillators with different time scales and $C 12=0.5$. (a) Bifurcation diagram showing extrema of Ex1 in oscillator 1 as a function of $C 21$. (b1) and (b2) are time series detail for $C 21 \approx 1.63$, and (c1) is the three-dimensional state space portraits with EX1, IN1 and the ultraslow population. (b3), (b4), and $(\mathrm{c} 2)$ are the same for $\mathrm{C} 21 \approx 1.92$.

For $C 21 \approx 1.92$ [Figs. 5(b3), 5(b4), and 5(c2)], the oscillatory period starts with a long segment of small amplitude fast activity in oscillator 1 with a frequency that is faster than with $C 21 \approx 1.63$ above. It is accompanied by large amplitude oscillations in oscillator 2. Toward the end, this is followed by slowly modulated fast activity (as found with $C 21 \approx 1.63$ ). The amount of slow modulations [3 in
Fig. 5(b3)] varies from instance to instance. The state space portrait shows the long thin tunnel during the regular fast activity at the top of which lies on a torus-like topology.

Next, we increase $C 12$ to 1.5 and compare the outcomes at this stronger coupling with dissimilar time scale parameters. Figure 6 shows that the bifurcation diagram is qualitatively similar to the 
one in Fig. 5 in the sense that for increasing coupling C21, we can observe a transition from steady state to oscillatory and transient seizure-like behavior.

For $C 21 \approx 1.06$ [Figs. 6(b1), 6(b2), and 6(c1)], oscillator 1 demonstrates an onset of slow activity, while oscillator 2 displays large spikes at low frequency, which evolve into spike-and-wave activity. The periods of wave are fairly regular in the central section but there is slowing toward the end. The state space portrait of oscillator 1 forms a structure reminiscent of a kettlebell with the pseudo-steady state as a handle. The small spike component is visible only in the portrait of oscillator 2 (not shown).

For $C 21 \approx 1.09$ [Figs. 6(b3), 6(b4), and 6(c2)], there is an initial pseudo-steady period, followed by a small amplitude lowfrequency oscillation in both oscillators. This then evolves with increasing amplitude into large-slow waves (oscillator 1) and spikewaves (oscillator 2 ) similar to the behavior found at $C 21 \approx 1.06$. The state space portrait Fig. 6(c2) is qualitatively similar to the one in Fig. 5(c2) apart from the frequency of the oscillations.

For $C 21=2$ [Figs. 6(b5), 6(b6), and 6(c3)], there is a long initial (upper) pseudo-steady state in both oscillators followed by a step-like decrease and a single slow wave in oscillator 1 . The steplike decrease is followed by small amplitude fast activity in oscillator 2 . The former slow waves seen at $C 21 \approx 1.09$ are now replaced by a long period of silence in oscillator 1 . Due to the coupling between the oscillators, the fast oscillations of oscillator 2 can actually be seen under magnifications in the time series of oscillator 1 . The state space portrait of oscillator 1 is essentially one large limit cycle of the relaxation type [Fig. 6(c3)].

As $C 21$ is increased further [C21 =3, Figs. 6(b7), 6(b8), and $6(\mathrm{c} 4)$ ], the picture remains qualitatively similar. The main change is that following a long silent period and a step-like decrease, both oscillators display small-fast onset activity. The amplitude is larger in the (fast) oscillator 2. There is an increase in amplitude during the oscillatory period. Consequently, the state space portrait has a helical component with increasing diameter [Fig. 6(c4)].

\section{Response to pulse perturbation during the quiescent period}

The response to pulse perturbation has been suggested as a potential dynamic biomarker of the slow evolution of the interictal brain as it approaches seizure onset. ${ }^{27-30} \mathrm{We}$ have, therefore, implemented repetitive pulse perturbation of our model of spontaneous seizure onset. We add a periodic stimulus to one of the oscillators in the form of a perturbation of $h_{e x 1}$ for the model equation (2) with dissimilar time constants of the two oscillators. The modified $h_{e x 1}$ pulse perturbation with its formation $h_{e x 1}=h_{e x}+a m p$ $* H(\sin (2 \pi t) / p s m) *\left(1-H\left(\sin \left(2 \pi\left(t+d \_s m\right)\right) / p s m\right)\right)$, where $h_{e x}$ $=-0.55$ is the constant parameter. amp is the amplitude of a pulse with 0.5 for small-fast onset and 0.05 for large-slow onset. $H(x)$ is a Heaviside function with $x>0, H(x)=1$, else $H(x)=0$. $d_{-} s m=1$ is the duration of positive current per stimulation unit. $p s m=20$ is the duration of a pulse.

Figure 7 shows the time series and state space of both oscillators during the quiescent state of the dynamics when parameters are set in the bursting regime with a fast-small amplitude onset of oscillations. We find that the response is clearly visible and increases slightly with time in the stimulated oscillator [Fig. 7(a2)] but that there is a jump in amplitude after which the response remains large until seizure onset. The state space display of the trajectory [Fig. 7(b2)] shows the two phases as a small more harmonic cycle and a large anharmonic limit cycle. The response of the nonstimulated variable is initially a time series where the stimulation is hardly perceptible [Fig. 7(a1), left half] but shows a sudden increase to a large response prior to the seizure [Fig. 7(a1), right half]. The state space appears to switch from a noisy quasi-steady state to a quasi-periodic cycle. The jump in amplitude is also seen at lower frequencies of perturbation, ruling out a summative effect of consecutive pulses. However, it is only seen above a threshold amplitude of the perturbation indicating that the response needs to be large enough to enter the state space region of the larger pseudo-cycle.

In contrast, Fig. 8 shows the time series and state space of both oscillators during the quiescent state of the dynamics when parameters are set in the bursting regime with a slow-large amplitude spiking at the onset of oscillations. We find that the response is clearly visible and increases slightly with time in the stimulated oscillator until the seizure onset is reached [Fig. 8(a2)]. No jump of amplitude is observed. The state space display of the trajectory [Fig. 8(b2)] shows a noisy limit cycle with slow drift. The response of the non-stimulated variable is weak and irregular, implying that it mostly depends on the nature of the noise [Fig. 8(a1)]. The state space display of the trajectory [Fig. 8(b2)] shows a noisy steady state with slow drift. If we add pulse stimulation at parameter settings in the oscillatory region near the onset $C 21 \approx 0.5$ in Fig. 4 (the case reminiscent of interictal epileptiform discharges), we can easily entrain the discharges with repetitive low-frequency pulses. If we add pulse stimulation at parameter settings in the oscillatory region near the onset of bursting $C 21 \approx 1.3$ in Fig. 5 (quiescent state), we can induce either single large spikes or repetitive spikes reminiscent of clinical afterdischarges (Ads). Further simulations show that the observed jump in amplitude reported in Fig. 7 is not seen in the 3 variable oscillator equation (1). It is also not found in the model equation (2) if the time scales of the two oscillators are similar but as mentioned the parameter space is too high-dimensional to further generalize this result.

\section{DISCUSSION}

We investigate a dynamic model of spontaneous seizure generation in focal-onset epilepsies. Clinical epileptic seizures are typically accompanied by abnormal discharge patterns on the level of invasive electroencephalography (EEG) (ECoG and SEEG). These discharge patterns are commonly used (in conjunction with other data) to classify and diagnose the seizure type. However, classification is mostly descriptive due to the complexity of the dynamics involved. ${ }^{4,8,31}$ The development of quantitative methods to analyze seizure EEG (and other EEG abnormalities like interictal spikes) is rather inconsistent due to the lack of a mechanistic understanding of the dynamic nature of the transitions and turns out to be based on qualitatively different kinds of underlying mental models. These models range from purely statistical models (e.g., change of EEG variance) to neural firing models to differential equation models for a single macroscopic location within the brain to explicit dynamic 
(A)
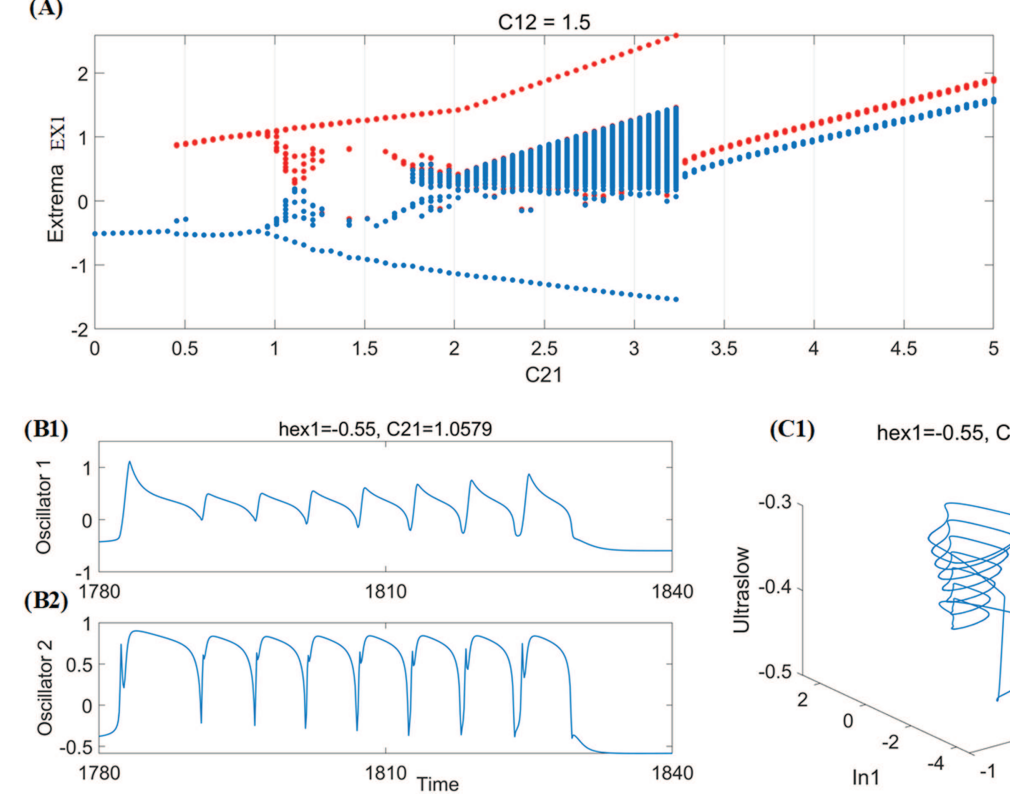

(B3)

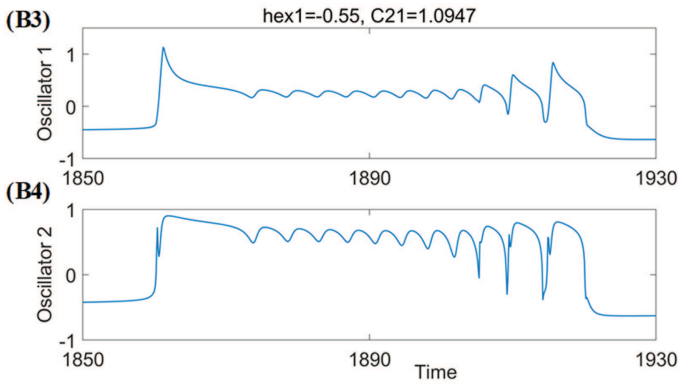

(B5)
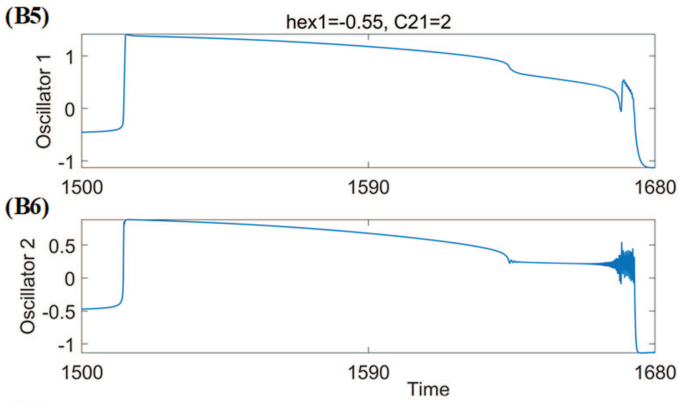

(B7)

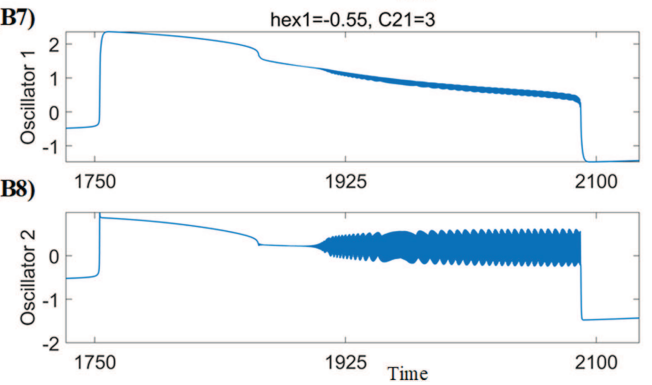

(C1) hex1=-0.55, C21 $=1.0579$

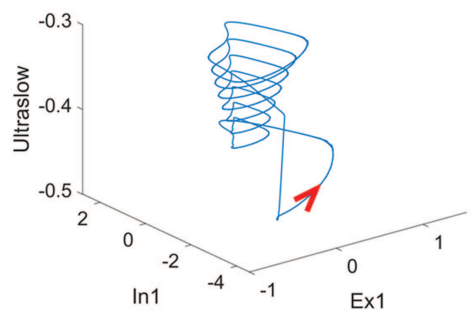

(C2) hex1=-0.55, C21 1.0947

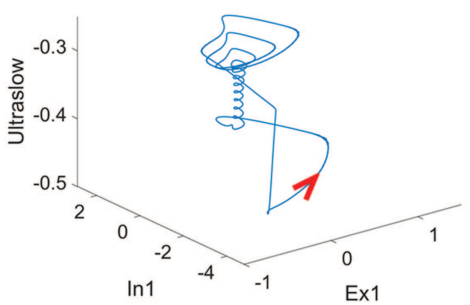

(C3) $\quad h e x 1=-0.55, C 21=2$

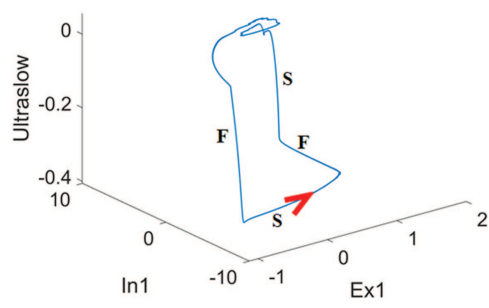

(C4) hex $1=-0.55, \mathrm{C} 21=3$

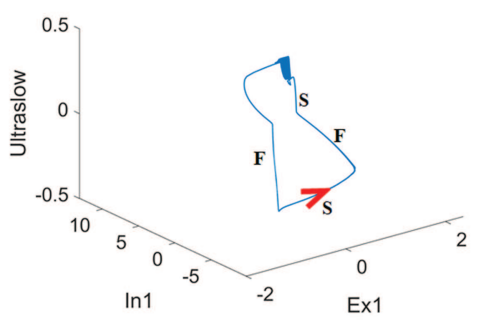

FIG. 6. Effect of $\mathrm{C} 21$ on model dynamics for different time scales and $C 12=1.5$. (a) Bifurcation diagram of extrema in oscillator 1 as a function of C21. (b1) and (b2) are the detailed time series for $\mathrm{C} 21 \approx 1.06$, and $(\mathrm{c} 1)$ is the three-dimensional state space portrait with EX1, IN1 and ultraslow population. (b3), (b4), and (c2) same for $\mathrm{C} 21 \approx 1.09$, (b5), (b6), and (c3) same for $C 21=2$, and (b7), (b8), and (c4) same for $\mathrm{C} 21=3$. 
(A1)

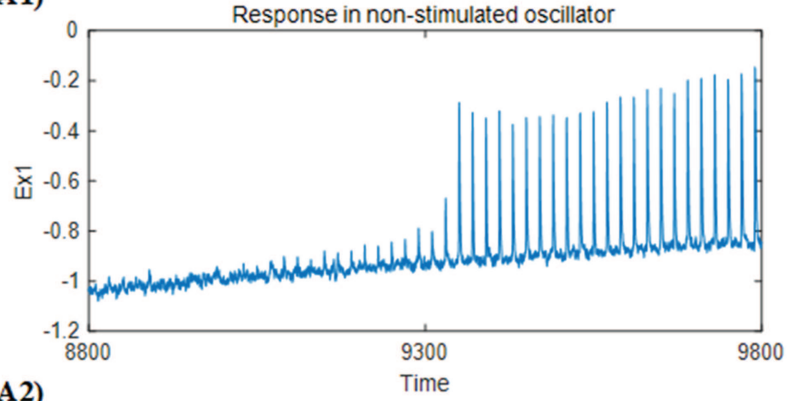

(A2)

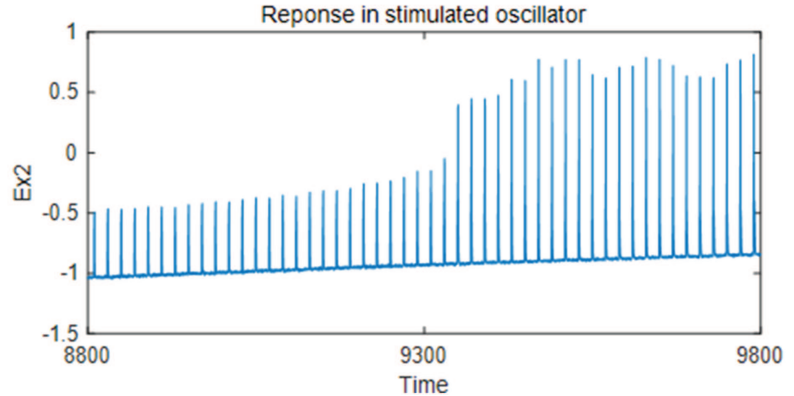

(B1)

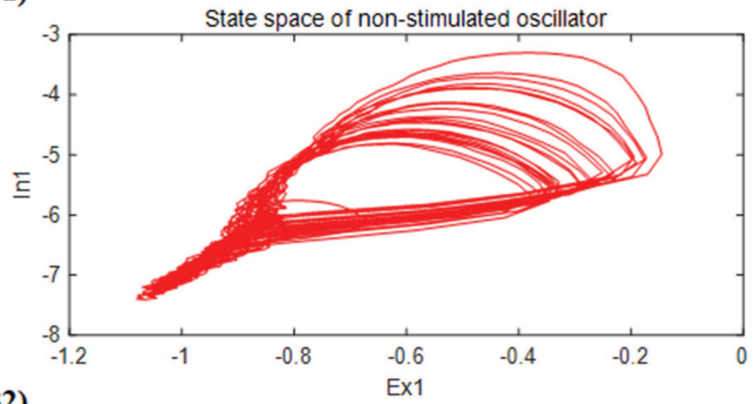

(B2)

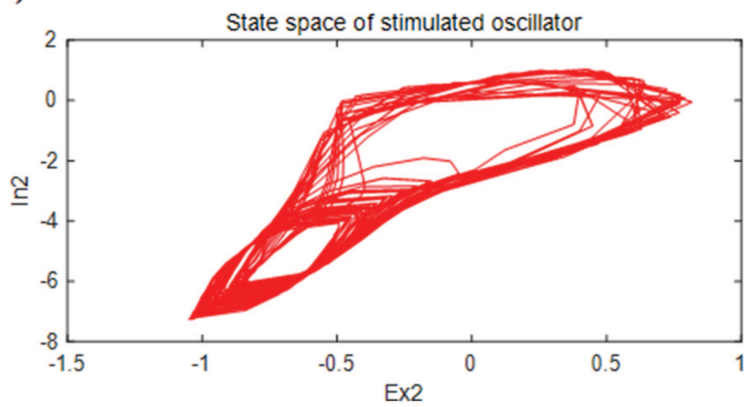

FIG. 7. Effect of periodic pulse stimulation of the quiescent state. Parameters as in Fig. 3(b1). Time series (a1) and state space (b1) of non-stimulated oscillator. Time series (a2) and state space (b2) of stimulated oscillator.

(A1)

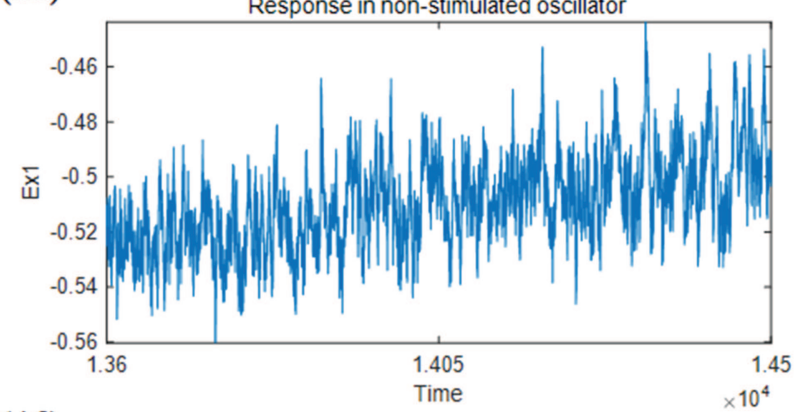

(A2)

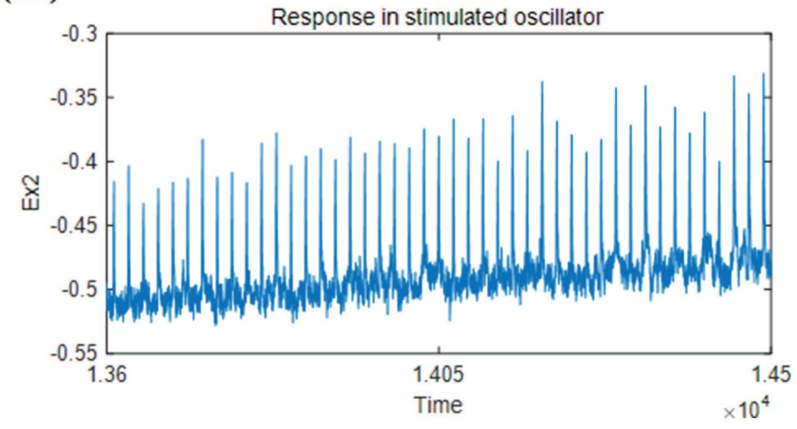

(B1)

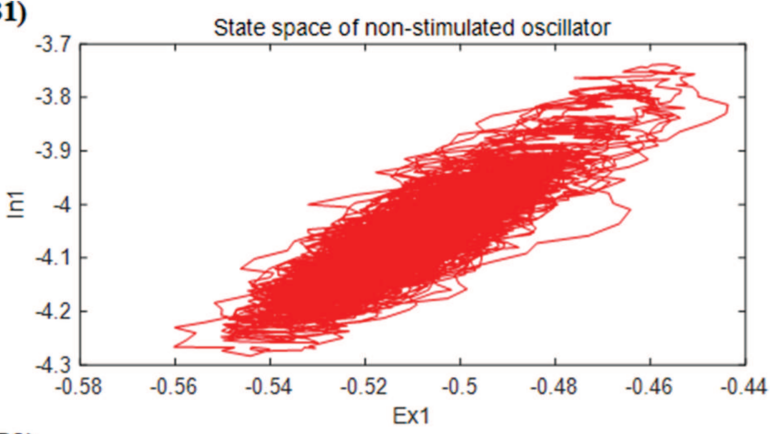

(B2)

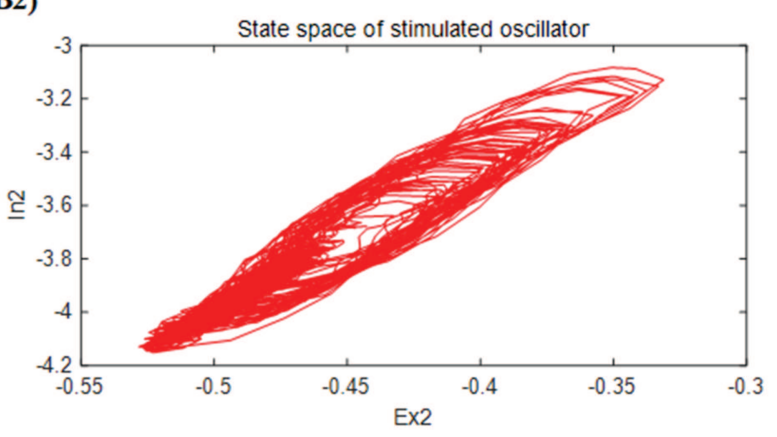

FIG. 8. Effect of periodic pulse stimulation of the quiescent state. Parameters as in Fig. 3(b2). Time series (a1) and state space (b1) of non-stimulated oscillator. Time series (a2) and state space (b2) of stimulated oscillator. 
network models covering those parts of the brain that contribute to EEG generation.

By introducing the three-variable model equation (1), we consider it a principal model of spontaneous transitions into and out of epileptiform seizure rhythms as routinely recorded, for example, in invasive recordings from patients with medically intractable epilepsy during pre-surgical evaluation. We use a mechanistic formalism based on the description of electric activity of neural populations. That is, the variables in the model describe the collective behavior of the large number of neurons that contribute to the recording of abnormal discharges and the environmental response in a local field potential. The advantage of such a model is the low number of variables as compared to, e.g., individual neural spiking models, while preserving the feature of being predictive (meaning they can be used to study the impact of drug treatment and electrical stimulation). From a dynamical point of view, the requirement is that the model operates near a transition from normal to abnormal activity, i.e., parameters are set near a suitable bifurcation point. Typically, models of that kind were studied by tuning parameters across the bifurcation but more recently approaches to spontaneous transitions have shown the dynamic mode of intermittency. In this case, the intrinsic dynamics is such that there are spontaneous transitions into and out of the abnormal rhythm. ${ }^{19,20}$

The minimal network model Eq. (2) describes two connected cortical oscillators under the control of one common slow time scale population. The prototypic $E X-I N$ circuit can be adjusted to display the major types of bifurcations between a fixed point to oscillatory dynamics. Because such an oscillator is a modeling construct and cannot properly be studied experimentally in isolation (out of the context of the whole brain), the clinically inferred bifurcation types are used to adjust the parameters. ${ }^{13,19,32}$ The oscillators are mutually connected in an excitatory fashion, assuming that the connection is dominated by populations of excitatory synapses. The connection is considered asymmetric in general, equal-weight bidirectional connection being singular and unrealistic. Each oscillator operates on a single time scale, which is a strong simplification. However, as multiple frequencies underlie epileptiform activity in general, we allow the time scales to be different in general. The final component of the model is an ultraslow population. Both oscillators feed into it and both oscillators receive feedback from it. This models a slowly varying process that during normal oscillator activity leads to a slow shift of conditions toward the transition to abnormal discharges. Various biophysical mechanisms have been proposed for this process but we refrain from an interpretation as neither has been conclusively confirmed in human epilepsy.

Mathematically, a spontaneous switching process is easy to implement using a single population with a slow time constant in conjunction with an oscillator if there exists a region of bistability. ${ }^{21}$ The complete model of five coupled variables then displays a wide bursting region in parameter space where there are spontaneous transitions between resting and oscillatory state. In many cases, the overall dynamics, while running on a low-dimensional attracting manifold, is irregular in the sense that durations of the periods and oscillatory details differ even in the absence of noise.

The construction of the dynamic bistability in the present model is only one of many possible ways. For instance, it has been reported that bistability can result from the introduction of time delays in models that do not possess bistability otherwise. ${ }^{33}$ The way forward here will be to design a set of models with alternative mechanisms that explain some aspects of the data and then design predictions that allow to distinguish between them, e.g., based on clinically implementable pulse stimulation protocols (see discussion of stimulation results below).

Our results focus on the focal onset and evolution of wave forms in order to compare the model output with known clinical seizure types. From Ref. 34, partial (focal, local) seizures are those in which, in general, the first clinical and EEG changes indicated initial activation of a system of neurons limited to part of one cerebral hemisphere. Generalized seizures are those in which the first clinical changes initial involvement of both hemispheres, while from Ref. 35, we know that the partial-onset seizures can become secondarily. But generalized-onset seizures are expected to remain generalized. The partial complex seizures emphasize the role of a spatially extended epileptic system that regulates the onset of both partial (focal, local) and generalized epileptic seizures. From Ref. 36, childhood absence epilepsy (CAE) is a familial type of epilepsy that exhibits spontaneous paroxysmal dynamics. From human observations, GABA mimetic anti-epileptic drugs exacerbate absence seizures. The typical EEG feature of CAE is quickly generalized spike-wave discharges (SWD) and the idea of seizure onset is that there is a transition between two attractors caused by intrinsic perturbations. Also, this paper pointed out that absence seizures in CAE have a focal cortical onset. From the view of our study, a slow variable or population has strong effects on the onset of focal seizures and thus, e.g., the effect of hyperventilation might be to modulate some ultraslow variable and thus lead to seizures indirectly. If various cortical locations are driven by a joint ultraslow variable, that might help to explain why a focal onset leads to rapid spreading of the abnormal activity. Thus, both focal and generalized onset could potentially employ features of our model.

Depending on fine-tuning of parameters within the region of intermittent dynamics, we can essentially model all known clinical seizure phenomenologies by tuning only a few model parameters. These include sinusoidal waves, nonlinear spikes, spike-waves, poly-spike waves, and all possible types of hybrids and transitions between types. For a basic clinical description of waveforms, see Ref. 37. The three main parameters considered (connectivity between oscillators and time scale ratio) in principle than allows fitting of these waveforms although this was not done in the current work. For the case of focal-onset seizures, a recent phenomenological classification of seizure types in invasive recording particularly highlighted the common types of small-amplitude fast oscillations and large amplitude slow spiking. ${ }^{8}$ This classification is important as it is connected, e.g., with clinical outcome of neurosurgery of the suspected epileptic focus. ${ }^{38}$ A previous modeling study using a detailed grid model of cortical oscillators investigated some characteristics of those types. ${ }^{7}$ Here, we find that the two types of seizure onset occur naturally as major spontaneous onset dynamics on the scale of a single cortical oscillator already (Fig. 3). Complex onset and evolution waveforms occur naturally on the two-oscillator model equation (2), e.g., the sharp waves (Fig. 5), spike-wave oscillations (Fig. 6), and poly-spike-waves (Fig. 5). Importantly, the model also generates a global transition to a silent state from which after some time oscillatory behavior, either fast-small oscillations or slow waves 
start the abnormal rhythmic discharge (Fig. 6). This is a long-known phenomenon in epileptic EEG, which to our knowledge has not yet been explained dynamically. Clinically, it is referred to as diffuse attenuation or diffuse electro-decrement. ${ }^{38}$ Here, we find it as a spontaneous transition to a global silent state from which individual oscillatory behavior arises depending on whether the underlying dynamic transition is akin to a supercritical Hopf or the SNIC type. Finally, the impact of the ultraslow population can also lead to transitions between dynamic states during one instance of abnormal rhythm resulting in complex dynamic seizure evolution. The most widely observed clinically is the tonic-clonic transition, which dynamically is a transition from simple oscillatory to burst-type oscillations [see, e.g., Fig. 5(b3)]. We also note that the nature of the model allows us to create hybrid behaviors between the basic discharge patterns. This allows for the interpretation of the so-called delta-brushes ${ }^{8}$ as a special case of burst oscillations.

The major clinical onset types (small-fast and large-slow onset) were immediately found in Eq. (2) based on parameter settings from the three-variable model equation (1). As such, we expect that this can be used as a starting point to construct larger networks with more oscillators to display the heterogeneity found in invasive clinical recordings. ${ }^{39}$ These include recruitment of different locations (nodes) at different times and to different degrees (i.e., the focality of onset); the observation of complex mixtures of waveforms during a single seizure; the observation of channels with different major frequency component during a single seizure; the finding that some locations drop out early of the seizure rhythm whereas others stop jointly; and others. Unfortunately, currently, no comprehensive mathematical theory of such transitions is available.

The fact that one model can simulate certain phenomenologies does not imply that it explains them. Different mechanisms might lead to similar waveforms and this calls for modeling to offer competing predictions of the same system. While experimentation is not permissible in human subjects, the response to stimulation done for clinical reasons in implanted patients offers an opportunity, particularly in the case of excitable systems.

In our network model, the inclusion of the common ultraslow population is the reason for the spontaneous dynamical transitions. ${ }^{19,20}$ In addition, we find that when the model is prepared in the steady state before this transition occurs [c.f. Fig. 4(a)], the steady state is excitable. This means that simulated pulse stimulation of the steady state can result in a single burst of abnormal activity either locally or globally. This is reminiscent of so-called afterdischarges in presurgical testing of cortical functionality. Afterdischarges (ADs) have long been suspected as markers of the ictogenic network but detailed studies of the findings in the context of clinical outcome have not been able to confirm the direct relationship of location of $\mathrm{AD}$ and the epileptic focus. Our studies indicate a complex relationship between response to pulse stimulation and involvement of a location in onset dynamics.

The presence or absence of the jump in response amplitude under stimulations (Figs. 7 and 8) shows that when changing from one ground state to another in the model, the state space structure changes qualitatively as well. Specifically, the situation in Fig. 8 demonstrates the presence of a region of exponential divergence in state space, which is not seen in Fig. 7. The characterization and modeling of such qualitative response differences might offer a criterion to distinguish between different model assumptions. Even for realistic models, this remains challenging, however, because the impact of local electrical stimulation is known to also impact distant sites but this impact only become visible in multimodel recording techniques, e.g., adding fMRI as done in non-human primates. ${ }^{40}$ Specifically, this calls for new techniques of dimensionality reduction when analyzing human invasive recordings, which give information about the most relevant state space features of the abnormal dynamics of a given patient.

If successful, the model allows predictive modeling of the response of epileptiform activity ( $\mathrm{AD}$ or seizure activity) to singlepulse stimulation with the goal of identifying most likely candidates for pulse stimulation to abort ADs or seizures. ${ }^{38}$ While we expect early versions of the current models to be too crude to make quantitative predictions, a model fit to clinical EEG during the period of implantation of a patient might allow for an adaptive predictive approach of responses to repeated stimulations. ${ }^{38,41}$ We propose that special attention be given to sudden changes of repeated stimulation responses either at the site of stimulation or at distant sites.

\section{ACKNOWLEDGMENTS}

This research was supported by the National Natural Science Foundation of China (NNSFC) (Grant Nos. 11932003 and 11772019). G.B. and L.Z. would like to thank Yujiang Wang, Peter Taylor, and Richard Rosch for discussion; and Beate Diehl for highlighting the role of single-pulse stimulation in neocortical dynamics.

\section{DATA AVAILABILITY}

The data that support the findings of this study are available from the corresponding author upon reasonable request.

\section{REFERENCES}

${ }^{1}$ A. T. Berg, S. F. Berkovic, M. J. Brodie et al., "Revised terminology and concepts for organization of seizures and epilepsies: Report of the ILAE commission on classification and terminology, 2005-2009," Epilepsia 51(4), 676-685 (2010).

${ }^{2}$ A. L. Velasco, C. L. Wilson, T. L. Babb et al., "Functional and anatomic correlates of two frequently observed temporal lobe seizure-onset patterns," Neural Plast. 7, 49-63 (2000).

${ }^{3}$ R. Wennberg, F. Arruda, L. F. Quesney et al., "Preeminence of extrahippocampal structures in the generation of mesial temporal seizures: Evidence from human depth electrode recordings," Epilepsia 43, 716-726 (2002).

${ }^{4}$ S. A. Lee, D. D. Spencer, and S. S. Spencer, "Intracranial EEG seizure-onset patterns in neocortical epilepsy," Epilepsia 41(3), 297-307 (2000).

${ }^{5} \mathrm{~J}$. A. Ogren, A. Bragin, C. L. Wilson et al., "Three-dimensional hippocampal atrophy maps distinguish two common temporal lobe seizure-onset patterns," Epilepsia 50(6), 1361-1370 (2009 Jun).

${ }^{6}$ I. Doležalová, M. Brázdil, M. Hermanová et al., "Intracranial EEG seizure onset patterns in unilateral temporal lobe epilepsy and their relationship to other variables," Clin. Neurophysiol. 124(6), 1079-1088 (2013 Jun).

${ }^{7}$ Y. Wang, A. J. Trevelyan, A. Valentin et al., "Mechanisms underlying different onset patterns of focal seizures," PLoS Comput. Biol. 13(5), e1005475 (2017).

${ }^{8}$ P. Perucca, F. Dubeau, and J. Gotman, "Intracranial electroencephalographic seizure-onset patterns: Effect of underlying pathology," Brain 137, 183-196 (2014).

${ }^{9}$ M. Stead, M. Bower, B. H. Brinkmann et al., "Microseizures and the spatiotemporal scales of human partial epilepsy,” Brain 133(9), 2789-2797 (2010).

${ }^{10}$ F. B. Wagner, E. N. Eskandar, G. R. Cosgrove et al., "Microscale spatiotemporal dynamics during neocortical propagation of human focal seizures," NeuroImage 122(11), 114-130 (2015). 
${ }^{11}$ S. Naze, C. Bernard, and V. K. Jirsa, "Computational modeling of seizure dynamics using coupled neuronal networks: Factors shaping epileptiform activity," PLoS Comput. Biol. 11(5), e1004209 (2015).

${ }^{12}$ F. Wendling, P. Benquet, F. Bartolomei et al., "Computational models of epileptiform activity," J. Neurosci. Methods 260, 233-251 (2016).

${ }^{13}$ Y. Wang, M. Goodfellow, P. N. Taylor et al., "Dynamic mechanisms of neocortical focal seizure onset," PLoS Comput. Biol. 10(8), e1003787 (2014).

${ }^{14}$ T. Proix, V. K. Jirsa, F. Bartolomei et al., "Predicting the spatiotemporal diversity of seizure propagation and termination in human focal epilepsy," Nat. Commun. 9(1), 1088 (2018).

${ }^{15} \mathrm{D}$. T. Liley and M. Walsh, "The mesoscopic modeling of burst suppression during anesthesia," Front. Comput. Neurosci. 7, 46 (2013).

${ }^{16}$ D. Pinotsis, P. Robinson, P. Beim Graben et al., "Neural masses and fields: Modeling the dynamics of brain activity," Front. Comput. Neurosci. 8, 149 (2014).

${ }^{17}$ U. Lalo, O. Palygin, S. Rasooli-Nejad et al., "Exocytosis of ATP from astrocytes modulates phasic and tonic inhibition in the neocortex," PLoS Biol. 12(1), e1001747 (2014)

${ }^{18}$ Y. Buskila, A. Bellot-Saez, and W. J. Morley, "Generating brain waves, the power of astrocytes," Front. Neurosci. 13, 1125 (2019).

${ }^{19}$ V. K. Jirsa, W. C. Stacey, P. P. Quilichini et al., "On the nature of seizure dynamics," Brain 137(8), 2210-2230 (2014)

${ }^{20}$ G. Baier, R. Rosch, P. N. Taylor et al. Design Principle for a Population-Based Model of Epileptic Dynamics. Complexity and Synergetics (Springer, Cham, 2018), pp. 333-347.

${ }^{21}$ E. M. Izhikevich, Dynamical Systems in Neuroscience - the Geometry of Excitability and Bursting (The MIT Press, Cambridge, 2007).

${ }^{22} \mathrm{P}$. N. Taylor and G. Baier, "A spatially extended model for macroscopic spikewave discharges,” J. Comput. Neurosci. 31, 679-684 (2011).

${ }^{23} \mathrm{H}$. R. Wilson and J. D. Cowan, "Excitatory and inhibitory interactions in localized populations of model neurons," Biophys. J. 12(1), 1-24 (1972).

${ }^{24}$ M. A. Kramer and S. S. Cash, "Epilepsy as a disorder of cortical network organization," Neuroscientist 18(4), 360-372 (2012).

${ }^{25}$ L. D. Iasemidis, S. Sabesan, and L. Good, "A new look into epilepsy as a dynamical disorder: Seizure prediction, resetting and control," Encyclopedia Basic Epilepsy Res. 2009, 1295-1302.

${ }^{26}$ Y. Wang, P. N. Taylor, M. Goodfellow et al., "A phase space approach for modelling of epileptic dynamics," Phys. Rev. E 85(6), 061918 (2012).
${ }^{27}$ R. Liu, K. Aihara, and L. Chen, "Dynamical network biomarkers for identifying critical transitions and their driving networks of biologic processes," Quant. Biol. 1(2), 105-114 (2013).

${ }^{28}$ M. A. Dahlem, S. Rode, A. May et al., "Towards dynamical network biomarker in neuromodulation of episodic migraine," Transl. Neurosci. 4(3), 282 (2013).

${ }^{29}$ D. R. Freestone, L. Kuhlmann, D. B. Grayden et al., "Electrical probing of cortical excitability in patients with epilepsy," Epilepsy Behav. 22, S110-S118 (2011).

${ }^{30}$ R. Enatsu, Z. Piao, T. O'Connor et al., "Cortical excitability varies upon ictal onset patterns in neocortical epilepsy: A cortico-cortical evoked potential study," Clin. Neurophysiol. 123, 252-260 (2012).

${ }^{31}$ D. Jiménez-Jiménez, R. Nekkare, L. Flores et al., "Prognostic value of intracranial seizure onset patterns for surgical outcome of the treatment of epilepsy," Clin. Neurophysiol. 126(2), 257-267 (2015).

${ }^{32}$ M. A. Kramer, W. Truccolo, U. T. Eden et al., "Human seizures self-terminate across spatial scales via a critical transition," Proc. Natl. Acad. Sci. U.S.A. 109(51), 21116-21121 (2012).

${ }^{33}$ J. Foss and J. Milton, "Multistability in recurrent neural loops arising from delay,” J. Neurophysiol. 84(2), 975-985 (2000).

${ }^{34}$ commission on Classification and Terminology of the International League Against Epilepsy. Proposal for revised clinical and electroencephalographic classification of epileptic seizures. Epilepsia 22, 489-501 (1981).

${ }^{35}$ R. Williamson, S. Hanif, G. C. Mathews et al., "Generalized-onset seizures with secondary focal evolution,” Epilepsia 50(7), 1827-1832 (2009).

${ }^{36} \mathrm{~J}$. Milton, J. Wu, S. A. Campbell, and J. Belair, "Outgrowing neurological diseases microcircuits, conduction delay and childhood absence epilepsy," in Computational Neurology and Psychiatry (Springer, 2017), pp. 11-48.

${ }^{37}$ E. Niedermeyer, The Generalized Epilepsies: a Clinical Electroencephalographic Study (Charles C. Thomas, Springfield, IL, 1972).

${ }^{38}$ P. N. Taylor, Y. Wang, M. Goodfellow et al., "A computational study of stimulus driven epileptic seizure abatement,” PLoS One 9(12), e114316 (2014).

${ }^{39}$ L. Zhang, Q. Wang, and G. Baier, "Dynamical features of a focal epileptogenic network model for stimulation-based control,” IEEE Trans. Neural Syst. Rehabil. Eng. 28(8), 1856-1865 (2020).

${ }^{40}$ A. S. Tolias, F. Sultan, M. Augath et al., "Mapping cortical activity elicited with electrical microstimulation using FMRI in the macaque," Neuron. 48, 901-911 (2005).

${ }^{41}$ D. Fan, Y. Zheng, Z. Yang et al., "Improving control effects of absence seizures using single-pulse alternately resetting stimulation (SARS) of corticothalamic circuit,” Appl. Math. Mech. 9, 1287-1302 (2020). 